\title{
Radial Symmetry for an Electrostatic, a Capillarity and some Fully Nonlinear Overdetermined Problems on Exterior Domains
}

\author{
W. Reichel
}

\begin{abstract}
We consider two physically motivated problems: (1) Suppose the surface of a body in $\mathbb{R}^{2}$ or $\mathbb{R}^{3}$ is charged with a constant density. If the induced single-layer potential is constant inside the body, does it have to be a ball? (2) Suppose a straight solid cylinder of unknown cross-section is dipped into a large plain liquid reservoir. If the liquid rises to the same height on the cylinder wall, does the cylinder necessarily have circular cross-section? Both questions are answered with yes, and both problems are shown to be of the type
\end{abstract}

$$
\operatorname{div}(g(|\nabla u|) \nabla u)+f(u,|\nabla u|)=0 \text { in } \Omega, \quad u=\text { const, } \frac{\partial u}{\partial \nu}=\text { const on } \partial \Omega, \quad u=0 \text { at } \infty
$$

where $\partial_{u} f \leq 0$ and $\Omega=\mathbb{R}^{N} \backslash \bar{G}$ is the connected exterior of the smooth bounded domain $G$. The overdetermined nature of this possibly degenerate boundary value problem forces $\Omega$ to be radial. This is shown by a variant of the Alexandroff-Serrin method of moving hyperplanes, as recently developed for exterior domains by the author in [19]. The results extend to MongeAmpère equations.

Keywords: Overdetermined problems, exterior domains, radial symmetry, electrostatic condenser, capillary surfaces

AMS subject classiflcation: Primary $35 \mathrm{~J} 65$, secondary 31 B 20, 76 B 45

\section{Introduction}

In this paper the following physical statements are shown to be characterizations of balls (under suitable regularity conditions):

$A$ constant charge distribution on the surface of a body $G$ in $\mathbb{R}^{2}$ or $\mathbb{R}^{3}$ induces a potential, that is constant inside the body, if and only if the body is a sphere.

A solid cylinder, if dipped into a large plain liquid reservoir, raises a capillary surface to a constant height on the outer cylinder wall if and only if the crosssection $G$ is a disk.

The mathematical reason for these physically intuitive statements is the fact, that constant Neumann and Dirichlet (i.e. overdetermined) boundary conditions in nonlinear

W. Reichel: Universität Karlsruhe, Mathematisches Institut I, D - 76128 
elliptic boundary value problems force the underlying domain to be radial. This observation is not new, e.g. see Serrin [20]; and has been shown to apply to a wide class of boundary value problems as'long as the underlying domain is bounded.

Our main theorem concerns the overdetermined boundary value problem on the unbounded domain $\Omega=\mathbb{R}^{N} \backslash \bar{G}$, which is supposed to be the connected exterior of the bounded domain $G \subset \mathbb{R}^{N}$ :

$$
\begin{aligned}
& Q u+f(u,|\nabla u|)=0 \text { in } \Omega, \quad 0 \leq u<b \text { in } \Omega \\
& u=b=\text { const }, \quad \frac{\partial u}{\partial \nu}=\text { const } \leq 0 \text { on } \partial \Omega \\
& u=0 \text { at } \infty .
\end{aligned}
$$

If $f(u,|\nabla u|)$ is (weakly) decreasing in $u$, then the interior domain $G$ is shown to be a ball. Here the operator $Q$ stands for three different types of elliptic operators: (I) the locally uniformly elliptic operator in divergence form

$$
Q u=\operatorname{div}(g(|\nabla u|) \nabla u), \quad g \in C^{2}[0, \infty), \quad g(s),(g(s) s)^{\prime}>0 \text { for } s \geq 0,
$$

(II) it's degenerate counterpart allowing $g(s)$ to vanish or explode at $s=0$, e.g. $\Delta_{p} u=$ $\operatorname{div}\left(|\nabla u|^{p-2} \nabla u\right), p>1$, and finally (III) the Monge-Ampère Operator

$$
Q u=\operatorname{det}\left(D^{2} u\right)
$$

as an example for a fully nonlinear operator. In section 2 the electrostatic problem is introduced and in section 3 the capillary surface problem is treated. Both problems are shown to be special cases of the general overdetermined problem treated in section 4 .

In the case $Q u=\Delta u$ our main theorem was proved in an earlier paper [19], where a more general nonlinearity $f(u,|\nabla u|)$ was admitted, which was only required to decrease for small values of $u,|\nabla u|$. In the case considered here, where $f(u,|\nabla u|)$ is decreasing for all $u,|\nabla u|$, an important short-cut in the proof is possible. Our main goal is to present this more direct approach, in particular because the two physical problems fall into this category. Furthermore, it is precisely this restriction to nonlinearities decreasing in $u$, which allows us to include degenerate elliptic operators. Since in cases.(I), (III) our main theorem is valid if $f(u,|\nabla u|)$ is locally Lipschitz continuous in $u,|\nabla u|$ and decreasing in $u$ only for $u,|\nabla u|$ small (see also Remark 2 in Section 4), we conjecture that this is also true for the degenerate case (II).

We note the very recently Aftalion and Busca [1] have obtained a symmetry result for an overdetermined problem $\Delta u+f(\dot{u})=0$ on an exterior domain $\Omega$, where $u^{-\frac{n+2}{n-2}} f(u)$ is (weakly) decreasing in $u$, but $f \geq 0$ may well be increasing.

In Serrin's interior domain-case, the radial symmetry of the underlying domain $G$ in direction $\eta \in \mathbb{R}^{N}$ is established by moving a hyperplane $T_{\lambda}$ perpendicular to $\eta$ from right to left up to $\partial G$. By moving it further left, it starts to cut a right hand cap $\Sigma(\lambda)$ off $G$, see Figure 1. The right hand cap, at least for small displacements of the touching hyperplane, has the property that it reflects into $G$. Thus, the function $u(x)-u\left(x^{\lambda}\right)$, 
comparing the values of $u$ at $x$ and at the reflected point $x^{\lambda}$, is well defined for points $x$ in the right hand cap.

Serrin shows by comparison principles the positivity of the comparison function up to a critical position of the hyperplane. Using the overdetermined constant boundary conditions, Serrin proves that the comparison functions vanishes identically in the critical right-hand cap. From that, the symmetry of the domain follows.
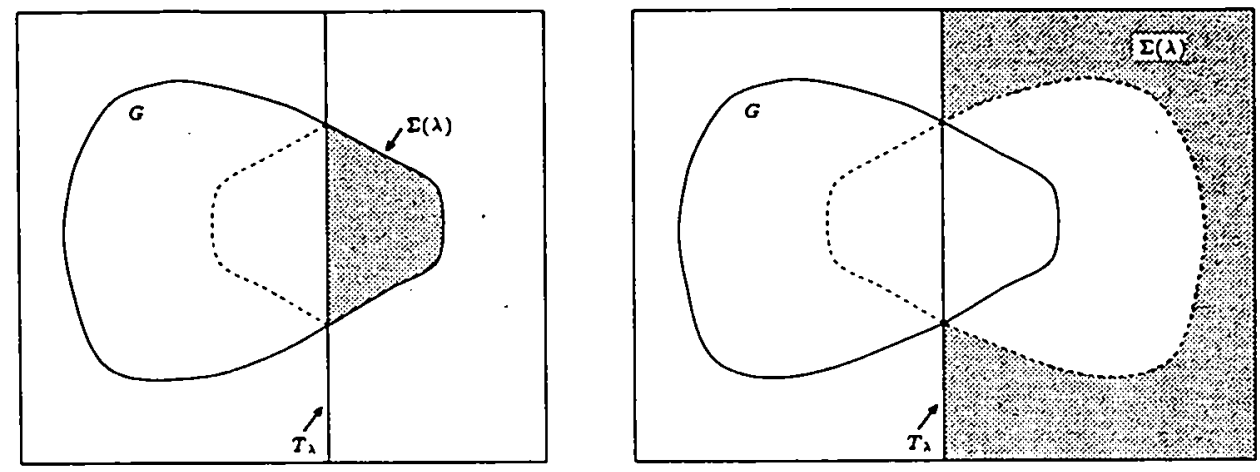

Figure 1: The right-hand cap in the interior and exterior case

For an exterior domain $\Omega=\mathbb{R}^{n} \backslash \bar{G}$, an analogous definition of a right-hand cap would be the set of all $x \in \Omega$, that belong to the right hand half-space of the hyperplane. But this procedure fails, since there are always points in this right-hand cap, that reflect into $G$ and, thus, do not admit the comparison $u(x)-u\left(x^{\lambda}\right)$. The novelty of our presentation is the definition of a right-hand cap suitable for exterior domains, see Figure 1.

For the locally uniformly elliptic and the Monge-Ampère operator classical strong and weak comparison principles are known and the treatment of these two operators differs only in technical details, i.e., the Monge-Ampère operator requires extra work.

The treatment of the degenerate elliptic operator is more delicate; in fact only few symmetry results for such operators are known, $[2,3,5,6,10,14]$. The difficulty lies in the loss of uniform ellipticity at points $x$ with $\nabla u(x)=0$ and, as a consequence, in the lack of a strong comparison theorem; for a counterexample see Walter [22]. For an interior overdetermined equation, this problem is avoided in Colesanti [6] by the hypothesis $\nabla u \neq 0$ in $\Omega$ with the possible exception of one point - the symmetry center. In our case we do not need to impose this assumption since we suppose $f(u,|\nabla u|)$ to be decreasing in $u$. This allows an important shortcut in the method of moving planes and admits us to use a strong comparison theorem only "close to the boundary of $\Omega$ ", as originally found by Alessandrini, Garofalo [3] and as used in Alessandrini [2] in the treatment of on overdetermined problem on annulus-type domains.

We use the following terminology: a real-valued function $h$, defined on an interval is called decreasing or weakly decreasing if $s \leq t$ implies $h(s) \geq h(t)$. It is called strictly decreasing if $s<t$ implies $h(s)>h(t)$. 


\section{The electrostatic problem}

We consider a bounded $C^{2, \alpha}$-domain $G \subset \mathbb{R}^{N}(N=2$ or $N=3)$ with connected exterior $\Omega=\mathbb{R}^{N} \backslash \bar{G}$. A charge distribution $\rho \in C(\partial \Omega)$ is called an equilibrium charge distribution if the induced single layer potential

$$
\Psi(x)=\int_{\partial G} \rho(y) \gamma(|x-y|) d \sigma_{y}
$$

is constant in $G$. Here $\gamma(r)=-\frac{1}{2 \pi} \log r$ for $N=2$ and $\gamma(r)=\frac{1}{4 \pi r}$ for $N=3$. An equivalent definition is given by the variational formulation to minimize the energy

$$
E(\tilde{\rho})=\int_{\partial G} \tilde{\rho}(x) \tilde{\Psi}(x) d \sigma_{x}=\int_{\partial G} \int_{\partial G} \tilde{\rho}(x) \tilde{\rho}(y) \gamma(|x-y|) d \sigma_{x} d \sigma_{y}
$$

among all $\tilde{\rho} \in C(\partial G)$ with (non-vanishing) constant total charge $\int_{\partial G} \tilde{\rho}(x) d \sigma_{x}$. We have the following statement:

Only the ball admits a constant equilibrium distribution.

The result is known for $N=2$ (see Martensen [15]). For $N=3$ it was conjectured by P. Gruber (see Heil and Martini [13: p. 353]).

The potential $\Psi$ is harmonic in $\mathbb{R}^{N} \backslash \partial G$ and both in $C^{2, \alpha}(\bar{G})$ and in $C^{2, \alpha}(\bar{\Omega})$ by the $C^{2, \alpha}$-regularity of the boundary. Suppose $G$ is a ball and $\rho$ is constant. Then it is easy to show that $(2.1)$ is invariant under rotations, and since the only rotationally invariant and smooth harmonic functions are constants, we see that balls do admit constant equilibrium distributions.

Now suppose a general domain $G$ with connected exterior admits a constant equilibrium distribution $\rho>0$. Then $\frac{\partial \Psi}{\partial \nu}=-\rho$ by the jump condition for single layer potentials ( $\nu$ always denotes the exterior normal to $G$ ). Furthermore, $\Psi \rightarrow \Psi_{\infty}$ at $\infty$ with $\Psi_{\infty}=-\infty$ for $N=2$ and $\Psi_{\infty}=0$ for $N=3$. In $\Omega$ we have $\Psi_{\infty}<\Psi<\Psi_{\partial G}$. The first inequality is obvious for $N=2$ and follows from (2.1) for $N=3$. Suppose $\Psi$ attains in $\Omega$ a value greater or equal $\Psi_{\partial G}$. Then $\Psi$ attains its maximum in $\Omega$. Since $\Omega$ is connected the maximum principle forces $\Psi$ to be constant contradicting the Neumann condition $\frac{\partial \Psi}{\partial \nu}<0$ on $\partial G$. Hence $\Psi$ satisfies the overdetermined boundary value problem

$$
\begin{aligned}
& \Delta u=0 \text { in } \bar{\Omega}, \quad u_{\infty}<u<u_{\partial \Omega} \text { in } \Omega \\
& u=\text { const }=u_{\partial \Omega}, \quad \frac{\partial u}{\partial \nu}=\text { const }<0 \text { on } \partial \Omega \\
& u=u_{\infty} \text { at } \infty
\end{aligned}
$$

where $u_{\infty}=-\infty$ for $N=2$ and $u_{\infty}=0$ for $N=3$. In Section 4 we shall show the radial symmetry of $\Omega$ for this type of problems. We note that the same result holds for $N>3$ without any changes.

We remark, that our problem is complementary to a problem studied by Philippin [16]. He studies an annulus type condenser $\Omega=\Omega_{0} \backslash \bar{\Omega}_{1}$ with separately starshaped domains $\bar{\Omega}_{1} \subset \Omega_{0}$. Here an equilibrium distribution on $\partial \Omega$ means that the single layer potential is 0 outside $\Omega_{0}$ and constant in $\Omega_{1}$ or, equivalently, that the energy 
$\int_{\partial \Omega_{0} \cup \partial \Omega_{1}} \tilde{\rho}(x) \tilde{\Psi}(x) d \sigma_{x}$ is minimal under the constraints $\int_{\partial \Omega_{i}} \tilde{\rho}(x) d \sigma_{x}=c_{i}$ for $i=1,2$ with constants $c_{1}, c_{2} \neq 0$. Philippin proves, that the only annulus type condenser that admits a component-wise constant equilibrium charge distribution consists of two concentric spheres. For $N \geq 3$ our case can be viewed as a limiting case of Philippin's problem, where the outer boundary of the condenser is removed. It is open, whether Phillipin's method, based on best-possible maximum principles for the Laplacian and Rellich's identity, can be adopted to our exterior-domain problem. Since we have more general equations than $\Delta u=0$ in mind, we have not pursued Phillipin's approach. Because existence results for overdetermined problems are impossible, there is no way to approximate our problem by a sequence of overdetermined problems on increasing finite annulus-type condensers. Finally we note that treatments of annulus-type condensers are also found in Alessandrini [2], Willms, Gladwell and Siegel [23] and Reichel [18], where no starshapedness assumption for Philippin's problem is made.

\section{The capillarity problem}

Here we consider a straight solid cylinder with the simply connected bounded $C^{2, \alpha}$ domain $G \subset \mathbb{R}^{2}$ as cross-section. If the cylinder is dipped into a plane infinite (physically: sufficiently large) reservoir of a liquid with constant surface tension situated in a homogeneous (non vanishing) gravitational field, then the resulting capillary surface is governed by the following boundary value problem in $\Omega=\mathbb{R}^{2} \backslash \bar{G}$ :

$$
\begin{array}{ll}
\operatorname{div}(T u)=\kappa u & \text { in } \Omega, \quad T u=\frac{\nabla u}{\sqrt{1+|\nabla u|^{2}}} \\
\nu \cdot T u=-\cos \gamma \quad \text { on } \partial G .
\end{array}
$$

Here $\gamma$ is the contact angle of the liquid and the cylinder wall, $\kappa>0$ is the capillarity constant, $u$ is the height of the surface above the reference level 0 at $\infty$, and $\nu$ is the exterior normal to $G$. We only consider $\gamma \in\left(0, \frac{\pi}{2}\right)$, since $\gamma=\frac{\pi}{2}$ means $u \equiv 0$, and nothing can be inferred as to the shape of $G$. Also we have to exclude $\gamma=0$ because of the regularity requirements, see Theorem 1 . The case $\gamma \in\left(\frac{\pi}{2}, \pi\right)$ can be transformed into the first problem by replacing $u$ with $-u$. We have the following statement:

The cross-section $G$ of the cylinder is a disk if and only if the liquid rises

to the same height on the whole cylinder wall.

The corresponding interior capillarity problem was studied by Serrin in a paper [20] that was most influential for the development of the moving plane device. A variant of Serrin's method is used in our proof. If $G$ is a disk, then the solution $u$ is rotationally symmetric by uniqueness, and therefore the liquid rises to the same level. The uniqueness and comparison theorem for exterior capillary surface problems is given in Appendix 2.

Now suppose $G$ is simply connected and $u$ rises to a constant level on the cylinder wall. It is known that $u$ is positive and tends to 0 at $\infty$ (see Appendix 2). By the strong minimum principle for nonlinear uniformly elliptic equations (see Protter and Weinberger (17: Section 16]) $u$ attains its maximum only on the boundary. Because 
of the constant Dirichlet boundary values we have $\nabla u=\nu \partial_{\nu} u$, and from that and the boundary condition for the normal derivative we obtain $\frac{\partial u}{\partial \nu}=\operatorname{const}=-\cot \gamma$ on $\partial G$. Finally, by the $C^{2, \alpha}$-regularity of $\partial G$ every solution $u \in C^{2}(\Omega) \cap C(\bar{\Omega})$ with bounded gradient (here we use $\gamma \neq 0$ ) belongs in fact to $C^{2}(\bar{\Omega})$ (see Gilbarg and Trudinger [12: Theorem 12.5, Remark (1)]). Summing up, we find that $u \in C^{2}(\bar{\Omega})$ satisfies the following overdetermined boundary value problem in $\Omega=\mathbb{R}^{2} \backslash \bar{G}$ :

$$
\begin{aligned}
& \operatorname{div}\left(\frac{\nabla u}{\sqrt{1+|\nabla u|^{2}}}\right)-\kappa u=0 \text { in } \bar{\Omega}, \quad 0<u<u_{\partial \Omega} \text { in } \Omega \\
& u=\text { const }=u_{\partial \Omega}>0, \quad \frac{\partial u}{\partial \nu}=\text { const }<0 \text { on } \partial \Omega \\
& u=0 \text { at } \infty .
\end{aligned}
$$

By the result of the following section, $\Omega$ turns out to be radial.

\section{The general result}

The two preceding results are special cases of the following general result. Let $G \subset \mathbb{R}^{N}$ be a bounded $C^{2, \alpha}$-domain such that $\Omega=\mathbb{R}^{N} \backslash \bar{G}$ is connected. We consider solutions $u$ of the problem

$$
\begin{aligned}
& Q u+f(u,|\nabla u|)=0 \text { in } \Omega, \quad 0 \leq u<b \text { in } \Omega \\
& u=b=\text { const }, \quad \frac{\partial u}{\partial \nu}=\text { const } \leq 0 \text { on } \partial \Omega \\
& u=0 \text { at } \infty
\end{aligned}
$$

under the following assumptions:

(I) Quasilinear, locally uniformly elliptic case

$(\mathrm{I})_{1} Q u=\operatorname{div}(g(|\nabla u|) \nabla u)$ with $g \in C^{2}[0, \infty), g(s)>0$ and $(g(s) s)^{\prime}>0$ for all $s \geq 0$.

$(\mathrm{I})_{2} f:[0, b] \times[0, \infty) \rightarrow \mathbb{R}$ is decreasing in its first variable and locally Lipschitz continuous on $[0, b] \times[0, \infty)$.

(I) $)_{3} u \in C^{2}(\bar{\Omega})$.

(II) Quasilinear, degenerate case

(II) $)_{1} Q u=\operatorname{div}(g(|\nabla u|) \nabla u)$ with $g \in C^{2}(0, \infty)$ and constants $k_{1}, k_{2}, k_{3}>0$ and $p>1$ such that $g(s) \geq k_{1} s^{p-2}, g(s)+s g^{\prime}(s) \geq k_{2} s^{p-2}$ and $g(s)+s\left|g^{\prime}(s)\right| \leq k_{3} s^{p-2}$ for all $s \geq 0$.

(II) $)_{2} f:[0, b] \rightarrow \mathbb{R}$ is independent on $\nabla u$, continuous, decreasing and Lipschitz continuous on $[0, b]$.

(II) $)_{3} u \in C^{1, \alpha}(\bar{\Omega})$. 
(III) Monge-A mpère case

(III) ${ }_{1} Q u=\operatorname{det}\left(D^{2} u\right)$.

$(\text { III })_{2} f:[0, b] \times[0, \infty) \rightarrow \mathbb{R}$ is decreasing in its first variable and locally Lipschitz continuous on $[0, b] \times[0, \infty)$.

$(\mathrm{III})_{3} u \in C^{3}(\bar{\Omega})$ is uniformly convex in every bounded subset of $\bar{\Omega}$.

Theorem 1. In the cases (I) - (III) the unique solution of problem (4.1) - (4.3) is radially symmetric, and the domain $G$ is a ball.

Remark 1. The electrostatic problem and the capillary surface problem are of type (I) with $g(s)=1$ and $g(s)=1 / \sqrt{1+s^{2}}$. The electrostatic problem for $N=2$ does not satisfy the boundary condition (4.3) $u=0$ at $\infty$. Nevertheless our method is still applicable, and the proof is given in [19].

Remark 2. For the quasilinear case (I) the above result is extended in [19] to more general functions $f(u,|\nabla u|)$, which are decreasing in $u$ only for $u,|\nabla u|$ small. For the Monge-Ampère case (III) such an extended result is also possible, whereas in the quasilinear degenerate case (II) this remains open due to the lack of a global strong comparison theorem.

Remark 3. If $G$ is bounded, open with finitely many components and its complement is connected, then Theorem 1 remains valid and $G$ is a ball. In this case we only require the Neumann boundary values to be constant on each component of the boundary, whereas the Dirichlet boundary values have to be the same for all boundary components.

4.1 Reflection in hyperplanes. Notation. By $x=\left(x_{1}, \ldots, x_{N}\right)=\left(x_{1}, x^{\prime}\right)$ we denote a point in $\mathbb{R}^{N}$ with $x^{\prime}=\left(x_{2}, \ldots, x_{N}\right) \in \mathbb{R}^{N-1}$, by $|x|$ its Euclidean norm and by $B_{r}(x)$ the open ball with center $x$ and radius $r$. For partial derivatives of a function $u$ we use $\partial_{x_{k}} u=\partial_{k} u=u_{k}$. It will be convenient to write $f=f(p, q)$ instead of $f\left(p, q_{1}, \ldots, q_{N}\right)$, where $q=\left|\left(q_{1}, \ldots, q_{N}\right)\right|$.

We shall show the symmetry of the domain $G$ and all solutions $u$ of problem (4.1) - (4.3) in the $x_{1}$-direction. Once this is done, the symmetry in any other direction and hence the radial symmetry follows by considering the function $u(M x)$, where $M$ is a rotation, as a solution of problem (4.1) - (4.3) on $M^{\top} \Omega$. For the $x_{1}$-symmetry we use the method of reflection in hyperplanes, which was first used by Alexandroff [4] and later refined and applied to nonlinear elliptic and parabolic partial differential equations by Serrin [20], Gidas, $\mathrm{Ni}$ and Nirenberg [11] and many others. Recently this method was modified and applied to unbounded domains by the author [19] and further extended by Aftalion, Busca [1].

We use the following definitions:

$$
\begin{array}{ll}
T_{\lambda}=\left\{x \mid x_{1}=\lambda\right\} & \text {-the hyperplane } \\
H_{\lambda}=\left\{x \mid x_{1}>\lambda\right\} & \text {-the right-hand half-space } \\
x^{\lambda}=\left(2 \lambda-x_{1}, x^{\prime}\right) & \text {-the reflection of } x \text { at } T_{\lambda}
\end{array}
$$




$$
\begin{array}{ll}
A^{\lambda}=\left\{x^{\lambda} \mid x \in A\right\} & \text {-the reflection of the set } A \text { at } T_{\lambda} \\
\Sigma_{1}(\lambda)=\left\{x \in G \mid x_{1}>\lambda\right\} & \text {-the inner right-hand cap } \\
\Gamma_{1}(\lambda)=\left\{x \in \partial G \mid x_{1}>\lambda\right\} & \text {-the inner right-hand boundary } \\
m_{1}=\sup \left\{x_{1} \mid x \in G\right\} & \text {-the } x_{1} \text {-extent of } G \\
\Sigma(\lambda)=H_{\lambda} \backslash \bar{G}^{\lambda} . & \text {-the reduced half-space. }
\end{array}
$$

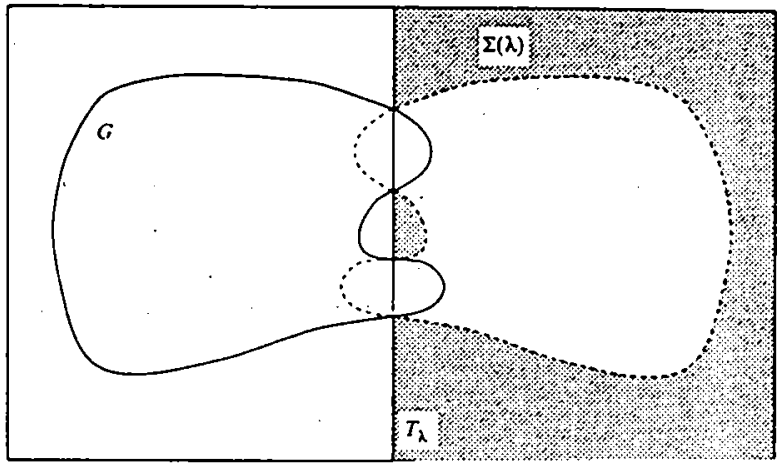

Figure 2: A reduced half-space with two components

Let us move the hyperplane into its critical position which is defined as follows: For $\lambda \in\left(m_{1}-\varepsilon, m_{1}\right)$ the reflection of $\Sigma_{1}(\lambda)$ lies inside $G$ and the positive $x_{1}$-direction is non-tangential on $\Gamma_{1}(\lambda)$. If we decrease $\lambda$, there will occur a critical value $m$ at which $\overline{\Sigma_{1}(\lambda)}$ meets $\partial G$ tangentially, or the normal on $\Gamma_{1}(\lambda)$ becomes orthogonal to the $x_{1}$-direction (see Figure 3 ).
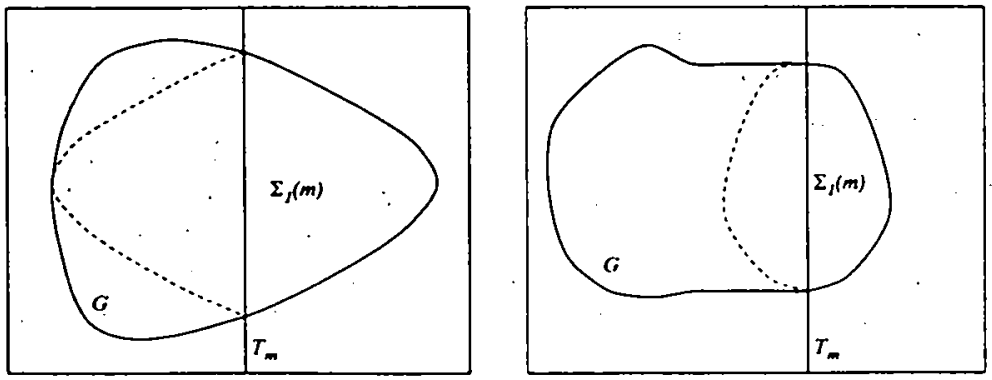

Figure 3: The critical positions of $T_{m}$

For this critical value $m$, let us define the comparison function

$$
w(x)=v(x)-u(x)=u\left(x^{m}\right)-u(x) \quad(x \in \overline{\Sigma(m)}) .
$$


Since the inner right-hand cap $\Sigma_{1}(m)$ reflects into $G$, we have $\Sigma_{1}(m) \subset G^{m}$. Therefore $x \in \Sigma(m)$ implies $x \notin \Sigma_{1}(m)$ and, by definition, $x^{m} \notin G$. Hence the comparison function is well defined on $\overline{\Sigma(m)}$. Note that $w=0$ on $\partial \Sigma(m) \cap T_{m}$ and $w=b-u \geq 0$ on $\partial \Sigma(m) \cap \partial G^{m}$, i.e., $w \geq 0$ on $\partial \Sigma(m)$. We shall show that in fact $w \equiv 0$, and from that the $x_{1}$-symmetry of $\Omega$ will be derived.

4.2 Proof of Theorem 1. Step 1: We shall show $w \geq 0$ in $\Sigma(m)$ and strong versions of this inequality by an elliptic comparison argument. First we note that $v(x)=u(2 m-$ $\left.x_{1}, x^{\prime}\right)$ satisfies the equation

$$
Q v+f(v,|\nabla v|)=0 \quad \text { on } \Omega^{m}
$$

For the quasilinear cases this follows from $\nabla v(x)=\left(-u_{1}\left(x^{m}\right), u_{2}\left(x^{m}\right), \ldots ; u_{N}\left(x^{m}\right)\right)$, but also the Monge-Ampère operator is invariant under isometric coordinate transformations. Furthermore in the Monge-Ampère case we note that $v$ and $\theta u+(1-\theta) v$ are locally uniformly convex on $\Sigma(m)$ for all $\theta \in[0,1]$.

In the quasilinear locally uniform case (I) we use the standard linearization

$$
\begin{aligned}
w_{t}(x) & =t v(x)+(1-t) u(x) \\
a_{i}(s) & =g(|s|) s_{i} \quad\left(s \in \mathbb{R}^{N}\right) \\
a_{i j}(x) & =\int_{0}^{1} \frac{\partial a_{i}}{\partial s_{j}}\left(\nabla w_{t}(x)\right) d t \\
b_{i}(x) & =\int_{0}^{1} \frac{\partial f}{\partial q_{i}}\left(w_{t}(x), \nabla w_{t}(x)\right) d t \\
c(x) & =\int_{0}^{1} \frac{\partial f}{\partial p}\left(w_{t}(x), \nabla w_{t}(x)\right) d t
\end{aligned}
$$

and obtain

$$
Q v-Q u+f(v, \nabla v)-f(u, \nabla u)=\partial_{i}\left(a_{i} ; w_{j}\right)+b_{i} w_{i}+c w=0 \quad \text { in } \Sigma(m) .
$$

In any bounded subset of $\Sigma(m)$ the coefficients $a_{i j}, \partial_{i} a_{i j}, b_{i}$ and $c \leq 0$ are bounded and the linearized operator is uniformly elliptic. Since $w \geq 0$ on $\partial \Sigma(m)$ and $w=0$ at $\infty$ we see by the strong maximum principle that $w$ cannot have a non-positive interior minimum in any component $Z$ of $\Sigma(m)$ unless it vanishes identically in $Z$. Hence either $w>0$ or $w \equiv 0$ in $Z$.

In the Monge-Ampère case (III) we define

$$
\begin{aligned}
D(s) & =\operatorname{det} s_{i j}, \quad s=\left(s_{i j}\right) \in \mathbb{R}^{N \times N} \\
M_{i j}(s) & =\frac{\partial D}{\partial s_{i j}}(s), \text { the co-factor of } s_{i j} \\
m_{i j}(x) & =\int_{0}^{1} M_{i j}\left(D^{2} w_{i}\right) d t
\end{aligned}
$$


and find the following linearization ( $b_{i}$ and $c$ as above):

$$
\operatorname{det} D^{2} v-\operatorname{det} D^{2} u+f(v, \nabla v)-f(u, \nabla u)=m_{i j} w_{i j}+b_{i} w_{i}+c w=0 \text { in } \Sigma(m) .
$$

Since $\left(M_{i j}\left(D^{2} w_{t}\right)\right)=\operatorname{det} D^{2} w_{t} \cdot\left(D^{2} w_{t}\right)^{-1}$ is uniformly positive definite in any bounded subset of $\Sigma(m)$ (here we need the locally uniform convexity of $w_{t}$ ), we have the locally uniform ellipticity of the above linearized operator. Therefore we have the same conclusion as before: In every component $Z$ of $\Sigma(m)$ either $w>0$ or $w \equiv 0$.

The degenerate quasilinear case (II) is different. First we define $v_{\varepsilon}(x)=v(x)+$ $\varepsilon(x \in \Sigma(m))$ and observe that $v_{\varepsilon}(x) \geq u(x)+\frac{\epsilon}{2}$ for $|x| \geq R$ and a suitably large $R$. In the remaining region $\Sigma_{R}=\Sigma(m) \cap B_{R}(0)$ we have $v_{\varepsilon} \geq u$ on $\partial \Sigma_{R}$, and we can use a weak comparison principle (see Tolksdorf [21]) for

$$
Q u+f(u)=0=Q v+f(v) \geq Q v_{\varepsilon}+f\left(v_{\varepsilon}\right) \quad \text { in } \Sigma_{R}
$$

to deduce $v_{e} \geq u$ in $\Sigma_{R}$. Letting $\varepsilon \rightarrow 0$ we obtain $v \geq u$ in $\Sigma(m)$. A strong comparison result as above is not available for degenerate quasilinear equations. Nevertheless we can do strong comparison "close to the boundary of $G$ " as in Alessandrini [2] and Alessandrini, Garofalo [3]. Let $Z$ be a component of $\Sigma(m)$. By the strong maximum principle and its boundary point version, which hold for (4.1) (see Tolksdorf [21: Propositions 3.2 .1 and 3.2.2]), the inequality $\nabla v \neq 0$ holds on $\partial \Omega^{m}=\partial G^{m}$, where $v$ attains its maximum, and remains true in a small open smooth neighbourhood $U$ of $\partial G^{m}$ in $\Sigma(m)$; we choose $U$ in such a way that $Z \cap U$ remains connected (see Figure 4). By local boundary regularity (see Fleckinger-Pellé, Takáć [9:Lemma 5.2]) we get $v \in C^{2}(\bar{U})$. Now we can use the strong comparison theorem of Tolksdorf [21: Theorem 3.3.2] to deduce $v>u$ or $v \equiv u$ in $Z \cap U$.

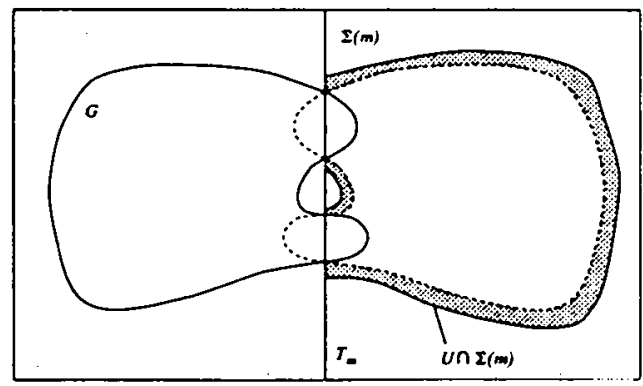

Figure 4: The choice of $U$

Step 2: Here we show that there is a component $Z$ of $\Sigma(m)$ with $w \equiv 0$ in $Z$ resp. $Z \cap U$, where in the degenerate case (II) $U$ is a suitable neighbourhood of $\partial G^{m}$. Suppose this were false, i.e. $w>0$ in $\Sigma(m)$ resp. $\Sigma(m) \cap U$. Recall that the critical point of $T_{m}$ originates either from (a) ${\overline{\Sigma_{1}(m)}}^{m}$ meeting $\partial G$ tangentially or from (b) the $x_{1}$-direction becoming tangent to $\partial \Sigma_{1}(m)$.

(a) Internal tangency: Let $q \in{\overline{\Sigma_{1}(m)}}^{m} \cap \partial G$ be the point where the reflected inner cap meets $\partial G$ tangentially, i.e., $q=p^{m}$ with $p \in \partial \Sigma_{1} \backslash T_{m}$. Since $w(p, m)=0$ we can use the Hopf lemma at the smooth boundary point $p \in \partial \Sigma(m)$ to deduce $\partial_{\nu(p)} w(p) \neq 0$; 
for the degenerate quasilinear case (II) see Tolksdorf [21: Theorem 3.3.1]. On the other hand $\nu(q)=\nu(p)^{m}$ by the internal tangency (here $\nu(q)$ is the common exterior normal of $\partial G$ and $\partial \Sigma(m)^{m}$ at $\left.q\right)$. Hence we calculate

$$
\frac{\partial v}{\partial \nu(p)}(p)=\frac{\partial u}{\partial \nu(q)}(q)=\text { const }=\left.\frac{\partial u}{\partial \nu}\right|_{\partial G}
$$

which results to $\partial_{\nu(p)} w(p)=0$ contradicting our previous statement.

(b) There is $p \in T_{m} \cap \partial G$ with $\nu_{1}(p)=0$ : At $p$ Hopf's lemma is no longer applicable since there is a right-angled corner of $\Sigma(m)$. Instead we show that $w$ has a second-order zero at $p$ in contradiction to Serrin's version of Hopf's lemma at corners (see Serrin [20] and Appendix 1). Recall that in the quasilinear degenerate case (II) the function $v$ is of class $C^{2}$ in a neighbourhood of $\partial \Omega^{m}$ and $u$ is $C^{2}$ in a neighbourhood of $\partial \Omega$ for the same reason. Hence we can carry out a $C^{2}$-analysis for $w$ close to the point $p$. Let us define a local coordinate frame with the $\xi_{n}$-axis along the normal $\nu(p)$ and the $\xi_{1}$-axis collinear to the $x_{1}$-axis. In this frame (see Figure 5) $\partial G$ is locally given by

$$
\xi_{N}=h\left(\xi_{1}, \ldots, \xi_{N-1}\right), \quad h(0)=0, \quad \nabla h(0)=0 \quad\left(h \in C^{2}\right)
$$

and the normal $\nu\left(\xi^{\prime}\right)$ at $\left(\xi^{\prime}, h\left(\xi^{\prime}\right)\right)$ is

$$
\nu\left(\xi^{\prime}\right)=\frac{\left(-\nabla h\left(\xi^{\prime}\right), 1\right)}{\sqrt{\left|\nabla h\left(\xi^{\prime}\right)\right|^{2}+1}}
$$

with $\nu(0)=(0, \ldots, 0,1) \in \mathbb{R}^{N}$. The new functions

$$
\tilde{u}(\xi)=u(x), \quad \tilde{v}(\xi)=v(x)=u\left(x^{m}\right), \quad \tilde{w}(\xi)=w(x)
$$

have the relations

$$
\begin{aligned}
\tilde{v}(\xi) & =\tilde{u}\left(-\xi_{1}, \xi_{2}, \ldots, \xi_{N}\right) \\
\tilde{w}(\xi) & =\tilde{u}\left(-\xi_{1}, \xi_{2}, \ldots, \xi_{N}\right)-\tilde{u}\left(\xi_{1}, \ldots, \xi_{N}\right) .
\end{aligned}
$$

With the help of the parametrisation $\left(\xi^{\prime}, h\left(\xi^{\prime}\right)\right)$ of $\partial G$ we can differentiate the constant Dirichlet and Neumann boundary conditions with respect to the tangent directions $\xi_{1}, \ldots, \xi_{N-1}$ and find

$$
\begin{aligned}
\tilde{u}_{j}(0) & =0 & & (j=1, \ldots, N-1) . \\
\tilde{u}_{N j}(0) & =0 . & & (j=1, \ldots, N-1) .
\end{aligned}
$$

Notice that $\tilde{w}_{\alpha}(0)=\tilde{w}_{\alpha \beta}(0)=\tilde{w}_{11}(0)=0$ for $\alpha, \beta \in\{2, \ldots, N\}$. Furthermore, $\tilde{w}_{1}(0)=$ $-2 u_{1}(0)=0$ by (4.7) and $\tilde{w}_{N_{1}}(0)=-2 \tilde{u}_{N_{1}}(0)=0$ by (4.8). For the second order zero of $\tilde{w}$ at $\xi=0$ it remains to calculate $\partial_{1 \alpha} \tilde{w}(0)$ for $\alpha=2, \ldots, N-1$. To do this we need the Taylor expansion of $\tilde{w}$ close to $\xi=0$

$$
\tilde{w}(\xi)=\sum_{\alpha=2}^{N-1} \partial_{1 \alpha} \tilde{w}(0) \xi_{1} \xi_{\alpha}+o\left(|\xi|^{2}\right) .
$$


Since $\tilde{w}>0$ for small values of $\xi_{1}>0, \xi_{\alpha} \in(-\varepsilon, \varepsilon)$ and $\xi_{N}>0$ we see that in fact $\partial_{1 \alpha} \tilde{w}(0)=0$. Hence $\tilde{w}$ and $w$ have a second-order zero at $p$ in contradiction to Serrin's corner lemma. This finishes the proof of Step 2 and shows that $w \equiv 0$ in a component $Z$ of $\Sigma(m)($ resp. $w \equiv 0$ in $Z \cap U)$.

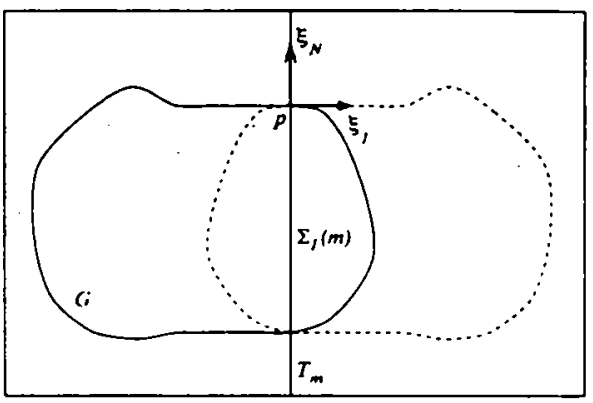

Figure 5: The $\xi$-coordinate system at $p$

Step 3: $w \equiv 0$ in $Z \cap U$ for a component $Z$ and a neighbourhood $U$ of $\partial G^{m}$ forces $\Omega$ to be symmetric. By the definition of $Z$ we find $\partial Z \backslash T_{m} \subset \partial G^{m}$. Since $w \equiv 0$ on $\partial Z$ we obtain $u \equiv b$ on $\partial Z \backslash T_{m}$ and hence $\partial Z \backslash T_{m} \subset \partial G$ by (4.1). The observation that $\partial Z \backslash T_{m}$ belongs both to $\partial G^{m}$ and to $\partial G$ is the essential reason for the symmetry of $G$. The following formal topological argument shows that $Z \cup Z^{m}$ extends across $T_{m}$ to an open subset of $\Omega$. Let

$$
X=Z \cup Z^{m} \cup(\partial Z \cap \Omega) \cup\left(\partial Z^{m} \cap \Omega\right) .
$$

We show that $X$ is open. Following from this we conclude

$$
\partial X \subset\left(\partial Z \cup \partial Z^{m}\right) \backslash(\partial Z \cap \Omega) \backslash\left(\partial Z^{m} \cap \Omega\right) \subset \partial \Omega
$$

which implies $\Omega=X$ since $X$ is non-empty and $\Omega$ is connected. To prove that $X$ is open we frequently use $\partial Z \backslash T_{m} \subset \partial G=\partial \Omega$ and $\partial Z^{m} \backslash T_{m} \subset \partial G=\partial \Omega$. Observe first that $Z \cup Z^{m} \subset$ int $X$.

(a) Take $x \in \partial Z \cap \Omega$. Since $\partial Z \backslash T_{m} \subset \partial \Omega$ we see that $x \in T_{m} \cap \Omega$. There exists a ball $B=B(x) \subset \Omega$, and we can define

$$
B_{>}=B \cap\left\{x_{1}>m\right\}, \quad B<=B \cap\left\{x_{1}<m\right\}, \quad E=B \cap\left\{x_{1}=m\right\} .
$$

Notice that $\partial Z \cap B_{>} \subset\left(\partial \Omega \cup T_{m}\right) \cap B_{>}=\emptyset$. Since $Z \cap B_{>} \neq \emptyset$ we get $B_{>} \subset Z$ and $B<\subset Z^{m}$. Together with $E \subset \Omega \cap\left(\bar{Z} \cap T_{m}\right) \subset \Omega \cap \partial Z$ this results in $B \cdot \subset X$, i.e. $x \in$ int $X$.

(b) Take $x \in \partial Z^{\dot{m}} \cap \Omega$. The proof of $x \in$ int $X$ works with notational changes as in part (a).

Remark. This last part of the proof goes back to lecture notes of L. E. Fraenkel (University of Bath, U.K.). 


\section{Appendix 1}

As an important extension of Hopf's maximum principle Serrin formulated in [20] the following lemma, which we quote for the reader's convenience.

Lemma. Let $D^{*}$ be a domain with $C^{2}$-boundary and let $T$ be a plane containing the normal to $\partial D^{*}$ at some point $p$. Let $D$ then denote the portion of $D^{*}$ lying on some particular side of $T$. Suppose that $w \in C^{2}(\bar{D})$ satisfies the elliptic differential equation

$$
\tilde{a}_{i j}(x) w_{i j}+\tilde{b}_{i}(x) w_{i} \leq 0 \quad \text { in } D
$$

where the coefficients are uniformly bounded. We assume that the matrix $\tilde{a}_{i j}$ is uniformly definite,

$$
\tilde{a}_{i j}(x) \xi_{i} \xi_{j} \geq k|\xi|^{2} \quad(k=\text { const }>0)
$$

and

$$
\left|\tilde{a}_{i j}(x) \xi_{i} \eta_{j}\right| \leq K(|\xi \cdot \eta|+|\xi| d(x)) \quad(K=\text { const }>0)
$$

where $\xi$ is an arbitrary vector in $I^{N}, \eta$ is the unit normal of the plane $T$ and $d(x)$ is the distance from $T$. Suppose also that $w \geq 0$ in $D$ and $w=0$ at $p$.

Then either

$$
\frac{\partial w}{\partial \mu}>0 \quad \text { or } \quad \frac{\partial^{2} w}{\partial \mu^{2}}>0 \quad \text { at } p
$$

unless $w \equiv 0$, where $\mu$ is any direction at $p$ which enters $D$ non-tangentially.

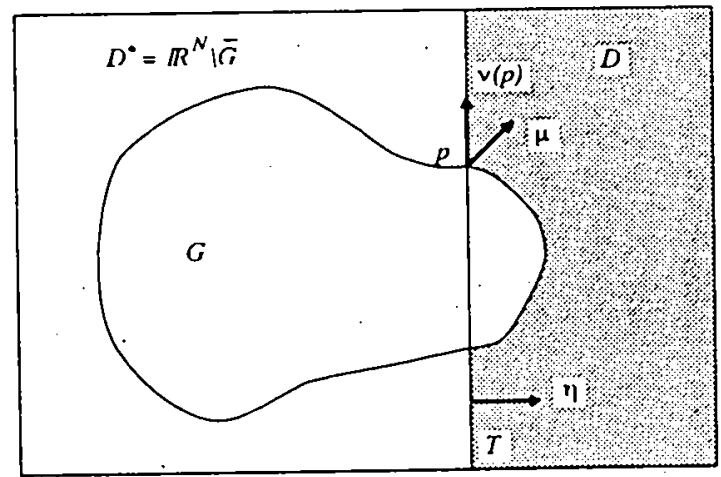

Figure 6: Serrin's lemma

We show how the lemma applies to our situation, where $T=T_{m}, D^{*}$ corresponds to $\mathbb{R}^{N} \backslash \bar{G}^{m}$ and $D$ to $\Sigma(m)$. Since the analysis is of local character at $p$, it is enough to verify the hypotheses in a neighbourhood of $p$, i.e. we intersect $D^{*}$ and $D$ with a small ball around $p$. Recall that both in the quasilinear locally uniform case (I) and in the Monge-Ampère case (III) $w$ satisfies a linear equation

$$
\tilde{a}_{i j} w_{i j}+\tilde{b}_{i} w_{i}+\tilde{c} w=0 \quad \text { in } D
$$

which is uniformly elliptic with bounded coefficients in every bounded subset of $\Sigma(m)$. 
For the quasilinear degenerate case (II) we found a neighbourhood $U$ of the point $p$ such that $u, v$ and $w$ are $C^{2}$-functions in $\overline{\Sigma(m) \cap U}$ with $\nabla u, \nabla v \neq 0$. Knowing this we can use the same linearization argument as in the locally uniform case (I) (see (4.5) and notice $\nabla w_{i} \neq 0$ on $\left.\overline{\Sigma(m) \cap U}\right)$ to obtain the linearized equation (A1.3) which is valid in $D=\Sigma(m) \cap U$.

With the standard substitution $\bar{w}=\exp \left(\beta x_{1}\right) w$ equation (A1.3) transforms into

$$
\tilde{a}_{i j} \bar{w}_{i j}+\bar{b}_{i} \bar{w}_{i}+\bar{c} \bar{w}=0 \quad \text { in } D
$$

where $\bar{b}_{i}=\tilde{b}_{i}-2 \beta \bar{a}_{1 i}$ and $\bar{c}=\tilde{a}_{11} \beta^{2}-\tilde{b}_{1} \beta+\tilde{c}$. In a neighbourhood of $p$ in $\Sigma(m)$, the operator $\tilde{a}_{i j} \partial_{i j}$ is uniformly elliptic, $\bar{b}_{i}$ is bounded and $\bar{c} \geq 0$ for a suitably large $\beta$, i.e.,

$$
\tilde{a}_{i j} \bar{w}_{i j}+\bar{b}_{i} \bar{w}_{i} \leq 0 \quad \text { in } D .
$$

It remains to verify (A1.2). For $x \in T_{m}$ the vectors $\nabla w$ and $\eta=(1,0, \ldots, 0)$ are collinear (recall $w=0$ on $T_{m}$ ), i.e., $\nabla w(x)=l(x) \eta$. We use the definition of $\tilde{a}_{i j}$ and find in the quasilinear cases (I) and (II) the following from (4.5) for $x \in T_{m}$

$$
\begin{aligned}
l(x) \tilde{a}_{i j}(x) \xi_{i} \eta_{j} & =(g(|\nabla v|) \nabla v-g(|\nabla u|) \nabla u) \cdot \xi \\
& =g(|\nabla u|)(\nabla v-\nabla u) \cdot \xi \\
& =l(x) g(|\nabla u|) \xi \cdot \eta .
\end{aligned}
$$

If $l(x) \neq 0$, the estimate $\left|\tilde{a}_{i j} \xi_{i} \eta_{j}\right| \leq K|\xi \cdot \eta|$ follows. For those $x \in T_{m}$ with $l(x)=0$ we find $0=\nabla w(x) \cdot \eta=2 \partial_{\eta} v(x)$. From the definition of $\tilde{a}_{i j}$ in (4.5) we get

$$
\left|\tilde{a}_{i j}(x) \xi_{i} \eta_{j}\right| \leq \int_{0}^{1}\left|\frac{g^{\prime}(|s|)}{|s|}(s \cdot \xi)(s \cdot \eta)\right| d t+\int_{0}^{1} g(|s|)|\xi \cdot \eta| d t=I_{1}+I_{2}
$$

with $s=t \nabla v(x)+(1-t) \nabla u(x)$, i.e., $s \cdot \eta=(2 t-1) \partial_{\eta} v=0$. So $I_{1}$ vanishes in those points $x \in T_{m}$, where $l(x)=0$, and $I_{2}$ is bounded above by $K|\xi \cdot \eta|$. Therefore we have

$$
\left|\tilde{a}_{i j}(x) \xi_{i} \eta_{j}\right| \leq K|\xi \cdot \eta| \quad\left(x \in T_{m}\right) .
$$

For the Monge-Ampère case (III) it is convenient to use $\eta=(1,0, \ldots, 0)$ and to calculate $\tilde{a}_{i 1}(x) \xi_{i}$ according to (4.6) for $x \in T_{m}$, where

$$
D^{2} w_{t}(x)=\left(\begin{array}{cccc}
u_{11} & (1-2 t) u_{12} & \ldots & (1-2 t) u_{1 N} \\
(1-2 t) u_{12} & u_{22} & \ldots & u_{2 N} \\
\vdots & \vdots & \vdots & \vdots \\
(1-2 t) u_{1 N} & u_{2 N} & \ldots & u_{N N}
\end{array}\right)
$$

For $\alpha=2, \ldots, N$ the co-factor $M_{\alpha 1}\left(D^{2} w_{t}\right)$ has the form $(1-2 t)$. co-factor of $D^{2} u(x)$. Hence $\tilde{a}_{\alpha 1}(x)=\int_{0}^{1} M_{\alpha 1}\left(D^{2} w_{t}\right) d t=0(\alpha=2, \ldots, N)$, and we have the estimate

$$
\left|\tilde{a}_{i j}(x) \xi_{i} \eta_{j}\right|=\left|\tilde{a}_{i 1}(x) \xi_{i}\right| \leq K\left|\xi_{1}\right|=K|\xi \cdot \eta| \quad\left(x \in T_{m}\right)
$$


which is the same estimate as in the quasilinear cases. By the smoothness of the expression $\tilde{a}_{i j}(x) \xi_{i} \eta_{j}$ in $x \in D$ we find

$$
\left|\tilde{a}_{i j}(x) \xi_{i} \eta_{j}\right| \leq K(|\xi \cdot \eta|+|\xi| d(x)) \quad(x \in D)
$$

where $d(x)$ is the distance of $x$ to $T_{m}$. Recall that we required $g \in C^{2}(0, \infty)$ in the quasilinear cases and $u \in C^{3}(\bar{\Omega})$ in the Monge-Ampère case, which gives $\tilde{a}_{i j}(x) \in C^{1}(\bar{D})$ (see (4.5) and (4.6)).

Since the hypotheses of Serrin's lemma are verified for (A1.5), we can conclude that $\bar{w}=\exp \left(\beta x_{1}\right) w$ does not have a second-order zero at $p$ unless it vanishes identically and, by a simple calculation, the same is true for $w$.

\section{Appendix 2}

The following theorem of Finn and Hwang [8] extends a well known comparison theorem for the capillary surface operator, see Finn [7: Theorem 5.1], to unbounded domains.

Theorem. Let $\kappa>0$ and suppose $\partial \Omega$ admits a decomposition $\partial \Omega=\Sigma^{\alpha} \cup \Sigma^{\beta}$, where $\Sigma^{\beta} \in C^{1}$ and $\nu$ is the interior normal to $\Omega$ on $\Sigma^{\beta}$. Let $u, v \in C^{2}(\Omega) \cap C^{1}(\bar{\Omega})$ with the properties

$$
\begin{aligned}
& \operatorname{div}(T u)-\kappa u \geq \operatorname{div}(T v)-\kappa v \text { for } x \in \Omega \text { such that } u>v \\
& u \leq v \text { on } \Sigma^{\alpha}, \quad \nu \cdot T u \geq \nu \cdot T v \text { on } \Sigma^{\beta}
\end{aligned}
$$

then $u \leq v$ follows. If the differential inequality holds everywhere in $\Omega$ and if $u(x)=v(x)$ for one $x \in \Omega$, then $u \equiv v$ results.

We draw two conclusions:

(1) The solution $u$ of the exterior capillary surface problem (as introduced in section 3 ) on the unbounded domain $\Omega=\mathbb{R}^{2} \backslash \bar{G}$ is unique.

(2) The solution $u$ is positive and tends to 0 at $\infty$.

Proof. (1) follows from the comparison theorem. (2) Since 0 is a subsolution, we obtain $u>0$ by the comparison principle. We assume $0 \in G$ and define $\varphi(r)=$ $\alpha e^{-\lambda r}(\alpha>0)$, where $\lambda$ is chosen so small that for sufficiently large $r=|x| \geq R_{0}$ we have

$$
\varphi^{\prime \prime}+\frac{\varphi^{\prime}}{r}=\left(\lambda^{2}-\frac{\lambda}{r}\right) \varphi \leq \kappa \varphi .
$$

It follows from the convexity of $\varphi(r)$ that

$$
\operatorname{div}(T \varphi)=\frac{\varphi^{\prime \prime}+\varphi^{\prime} / r}{\sqrt{1+\left|\varphi^{\prime}\right|^{2}}}-\frac{\left|\varphi^{\prime}\right|^{2} \varphi^{\prime \prime}}{\left(1+\left|\varphi^{\prime}\right|^{2}\right)^{3 / 2}} \leq \Delta \varphi \leq \kappa \varphi .
$$

Next we choose $\alpha>0$ such that $\varphi \geq u$ for $|x|=R_{0}$ and find by the above comparison principle $\varphi \geq u$ for $|x| \geq R_{0}$, i.e., $0<u=O(\exp (-\lambda r))$ as $r \rightarrow \infty$.

Acknowledgement. The author thanks the referee for valuable suggestions, which led to an improvement of this paper. 


\section{References}

[1] Aftalion, A. and J. Busca: Radial symmetry of overdetermined boundary value problems in exterior domains. Preprint.

[2] Alessandrini, G.: A symmetry theorem for condensers. Math. Meth. Appl. Sci. 15 (1992), $315-320$.

[3] Alessandrini, G. and N. Garofalo: Symmetry for degenerate parabolic equations. Arch. Rat. Mech. Anal. 108 (1989), 161 - 174.

[4] Alexandroff, A. D.: A characteristic property of the spheres. Ann. Mat. Pura Appl. 58 (1962), $303-354$.

[5] Badiale, M. and E. Nabana: A note on the radiality of solutions of the p-Laplacian equation. Applicable Anal. 52 (1994), 35 - 43.

(6) Colesanti, A.: A symmetry result for the p-Laplacion equation via the moving planes method. Applicable Anal. 55 (1994), 207 - 213.

[7] Finn, R.: Equilibrium Capillary Surfaces. New York: Springer-Verlag 1986.

[8] Finn, R. and J. Hwang: On the comparison principle for capillary surfaces. J. Fac. Sci. Univ. 'Tokyo 36 (1989), $131-134$.

[9] Fleckinger-Pellé, J. and P. Takáć: Uniqueness of positive solutions of nonlinear cooperative systems with the p-Laplacion. Indiana Univ. Math. J. 43 (1994), 1227 - 1253.

[10] Garofalo, N. and J. Lewis: A symmetry result related to some overdetermined boundary value problems. American J. of Math. 111 (1989), 9 - 33.

[11] Gidas, B., Wei-Ming $\mathrm{Ni}$ and L. Nirenberg: Symmetry and related problems via the maximum principle. Comm. Math. Phys. 68 (1979), $209-243$.

[12] Gilbarg, D. and N. S. Trudinger: Elliptic Partial Differential Equations of Second Order. 2nd Edition. New York: Springer-Verlag 1983.

[13] Heil, E. and H. Martini: Special convex bodies. In: Handbook of Convex Geometry (eds.: P. Gruber and J. Wills). Amsterdam: Elsevier 1993, pp. 347 - 385.

[14] Kesavan, S. and F. Pacella: Symmetry of positive solutions of a quasilinear elliptic equation via isoperimetric inequalities. Applicable Anal. 54 (1994), 27 - 37.

[15] Martensen, E.: Eine Integralgleichung für die log. Gleichgewichtsverteilung und die Krümmung der Randkurve eines ebenen Gebiets. Z. angew. Math. Mech. (ZAMM) 72 (1992), T596 - T599.

[16] Philippin, G.A.: On a free boundary problem in electrostatics. Math. Meth. Appl. Sci. $12(1990), 387-392$.

[17] Protter, M. and H. F. Weinberger: Maximum Principles in Differential Equations. New York: Springer-Verlag 1984.

[18] Reichel, W.: Radial symmetry by moving planes for semilinear elliptic BVPs on annuli and other non-convex domains. In: Progress in Partial Differential Equations: Elliptic and Parabolic Problems (Pitman Res. Notes: Vol. 325; eds.: C. Bandle et al). Harlow: Longman 1995, pp. $164-182$.

[19] Reichel, W.: Radial symmetry for elliptic boundary value problems on exterior domains. To appear in Arch. Rat. Mech. Anal.

[20] Serrin, J.: A symmetry theorem in potential theory. Arch. Rat: Mech. Anal. 43 (1971), $304-318$.

[21] Tolksdorf, P.: On the Dirichlet problem for quasilinear equations in domains with conical boundary points. Comm. Part. Diff. Equ. 8 (1983), $773-817$. 
[22] Walter, W.: A new approach to minimum and comparison principles for nonlinear ordinary differential operators of second order. Nonlinear Analysis, TMA 25 (1995), 1071 1078.

[23] Willms, N. B., Gladwell, G. and D. Siegel: Symmetry theorems for some overdetermined boundary value problems on ring domains. Z. Angew. Math. Phys. 45 (1994), 556 - 579.

Received 02.10.1995 